TITLE:

\title{
Visualizing water molecule distribution by atomic force microscopy
}

\section{$\operatorname{AUTHOR}(\mathrm{S})$ :}

Kimura, Kenjiro; Ido, Shinichiro; Oyabu, Noriaki; Kobayashi, Kei; Hirata, Yoshiki; Imai, Takashi; Yamada, Hirofumi

\section{CITATION:}

Kimura, Kenjiro ...[et al]. Visualizing water molecule distribution by atomic force microscopy. JOURNAL OF CHEMICAL PHYSICS 2010, 132(19): 194705.

\section{ISSUE DATE:}

2010-05

URL:

http://hdl.handle.net/2433/147208

\section{RIGHT:}

Copyright 2010 American Institute of Physics. This article may be downloaded for personal use only. Any other use requires prior permission of the author and the American Institute of Physics. The following article appeared in JOURNAL OF CHEMICAL PHYSICS132, 194705 (2010) and may be found at 


\title{
Visualizing water molecule distribution by atomic force microscopy
}

\author{
Kenjiro Kimura, ${ }^{1, a)}$ Shinichiro Ido, ${ }^{1}$ Noriaki Oyabu, ${ }^{1}$ Kei Kobayashi, ${ }^{2}$ Yoshiki Hirata, ${ }^{3}$ \\ Takashi Imai, ${ }^{4}$ and Hirofumi Yamada ${ }^{1}$ \\ ${ }^{1}$ Department of Electronic Science and Engineering, Kyoto University, Katsura, Nishikyo, \\ Kyoto 615-8510, Japan \\ ${ }^{2}$ Innovative Collaboration Center (ICC), Kyoto University, Katsura, Nishikyo, Kyoto 615-8520, Japan \\ ${ }^{3}$ National Institute of Advanced Industrial Science and Technology, 1-1-1 Umezono, Tsukuba, \\ Ibaraki 305-8568, Japan \\ ${ }^{4}$ Computational Science Research Program, RIKEN, 2-1 Hirosawa, Wako, Saitama 351-0198, Japan
}

(Received 16 December 2009; accepted 3 April 2010; published online 21 May 2010)

\begin{abstract}
Hydration structures at biomolecular surfaces are essential for understanding the mechanisms of the various biofunctions and stability of biomolecules. Here, we demonstrate the measurement of local hydration structures using an atomic force microscopy system equipped with a low-noise deflection sensor. We applied this method to the analysis of the muscovite mica/water interface and succeeded in visualizing a hydration structure that is site-specific on a crystal. Furthermore, at the biomolecule/ buffer solution interface, we found surface hydration layers that are more packed than those at the muscovite mica/water interface. () 2010 American Institute of Physics. [doi:10.1063/1.3408289]
\end{abstract}

\section{INTRODUCTION}

Hydration structures at biomolecule/liquid interfaces are closely related to highly ordered structures and biofunctions. For example, the highly ordered structure of DNA molecules is strongly dependent on relative humidity. ${ }^{1}$ In enzyme molecules, water molecules have an important role in stabilizing their active site structure with hydrogen combinations. ${ }^{2}$

In analyzing 3D hydration structures which mean steric water molecule distribution around atoms or molecules, neutron diffraction analysis is one of the most commonly used techniques because a large magnitude of neutron diffraction is observed at hydrogen atoms. However, this method requires a large crystal as a material, and crystallization is one of the limitations for applying this technique to the in situ measurement of hydration structures in various functional systems.

In theoretical chemistry, various molecular theories such as molecular dynamics (MD) simulation and the statisticalmechanical distribution function theory of liquids have revealed various microscopic pictures of solvation structures at the solid/liquid interface. For example, Akiyama and Hirata ${ }^{3}$ showed the dependence of the type of hydration structure on the configuration of surface atoms using the statisticalmechanical theory of molecular liquids known as the reference interaction site model (RISM) theory. Kovalenko and Hirata $^{4}$ succeeded in describing a 3D hydration structure on a metal surface by the 3D generalization of the RISM (3DRISM) theory.

Atomic force microscopy (AFM) has been used to investigate the solid/liquid interface. Jarvis et al. reported that when the AFM tip is close to the solid surface at the solid/ liquid interface, the force measured by AFM oscillates with a period equal to the diameter of the liquid molecule. ${ }^{5} \mathrm{Re}$ cently, we have succeeded in developing frequency modula-

${ }^{a)}$ Electronic mail: kimura@gold.kobe-u.ac.jp. tion AFM (FM-AFM) with atomic or molecular resolution in liquid. ${ }^{6}$ This development opened the door to structural biology studies in physiologically relevant biological environments on the atomic or molecular scale. Furthermore, Fukuma et al. reported an impressive image of a hydrated surface as a topographic image obtained by FM-AFM with a low-noise optical head. ${ }^{7}$ Hofbauer et al. also showed clear images of each solvation layer. ${ }^{8}$ In these publications, no images of the intermediate structure between layers in a vertical direction were shown. In addition, cross-sectional structures of local hydration layers in the vertical direction ${ }^{9}$ have not been fully investigated yet.

In this study, we demonstrate the visualization of crosssectional hydration layers in the vertical direction against the solid surface with atomic resolution and show the relationship between force distribution and water-molecule density distribution at the interfaces calculated by 3D-RISM. Here, we adopt a muscovite mica/water system as the target system because it has been studied extensively. Among compounds with hydrophilic surfaces, the mica substrate is useful for removing toxic materials owing to its replacement and absorption capabilities. Industrially, it is used for removing silver iodide. Silver iodide works as the nucleus in cloud seeding. ${ }^{10,11}$ The hydration structure on a muscovite mica substrate is related to the swelling characteristics of mica as a viscosity improver and a mold lubricant among its other uses. To date, many groups have studied the various physical characteristics of hydration structures on the muscovite mica surface, and the existence of icelike structures has been predicted. ${ }^{12-15}$

However, no local hydration structures at specific crystal sites on mica surface have been fully investigated on the atomic or molecular scale by in situ measurement. Recently, using in situ measurement, Cheng et al. have shown the average vertical density profile of water molecules at the mica/ water interface by $\mathrm{x}$-ray crystal truncation rod (CTR) 


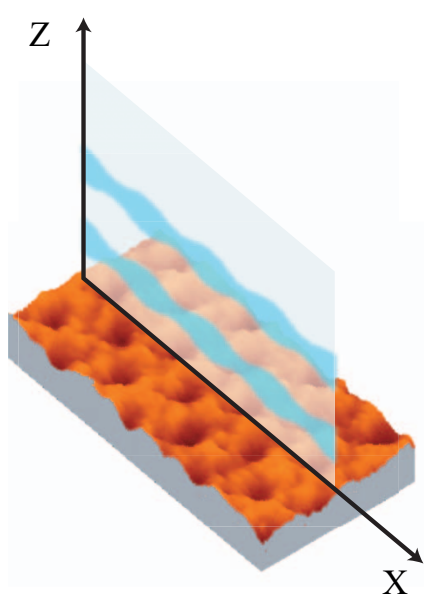

FIG. 1. Schematic illustration of hydration measurement by force mapping using AFM. In force mapping, the AFM tip is scanned one-dimensionally in the $Z$-direction while monitoring frequency shift $(\Delta f)$ related to interaction force $(f)$. After 1D scanning, the $X$-coordinate of the tip location is changed to the next line in the aqua area. By repeating these procedures, we can obtain a $2 \mathrm{D} \Delta f$ distribution.

scattering. ${ }^{16}$ The $\mathrm{x}$-ray CTR scattering extending in the direction perpendicular to the crystal surface provides structural information on the solid surface. Moreover, Park and Sposito ${ }^{17}$ calculated the water molecule distribution at the mica/water interface by MD simulation, and their results coincided with the density profile obtained by Cheng et al. ${ }^{16} \mathrm{In}$ this study, we focus on measuring local hydration structures in specific crystal sites with atomic resolution. In addition, we demonstrate the imaging of cross-sectional hydration structures above bacteriorhodopsin (bR), the protein constituent of purple membranes, to relate the application of our technique more closely to biological studies.

\section{EXPERIMENTAL AND THEORETICAL METHODS}

\section{A. Hydration measurement by FM-AFM}

Figure 1 shows a conceptual scheme of hydration measurement by force mapping using AFM. The $X$-axis is the surface direction, and the $Z$-axis is the vertical direction. The AFM tip is close to the surface from the $Z$-direction, and an interaction force-distance curve ( $F-Z$ curve) between the tip and the surface is measured. A 2D distribution of interaction forces is obtained by changing the $X$-coordinate of the $F-Z$ curve to the next position and repeating the $F-Z$ curve measurement. Then, the $2 \mathrm{D}$ distribution is pictured in $X Z$ plane, and the force is a function of $X$ coordinate and $Z$ one.

In $2 \mathrm{D}$ force mapping, we used an FM-AFM system with a low-noise deflection sensor. ${ }^{6}$ The noise level derived from the deflection sensor was about $20 \mathrm{fm} / \sqrt{\mathrm{Hz}}$, and the $Q$ factor at the first resonance of the cantilever was typically about 10 in water and $1 M \mathrm{KCl}$ aqueous solution. In the FM-AFM system, the cantilever was self-excited at its mechanical resonance frequency $\left(f_{0}\right)$, and its frequency was measured by an analog FM detector equipped with a voltagecontrolled xtal oscillator. ${ }^{18}$ In the experiment, $f_{0}$ was about $130 \mathrm{kHz}$. Vibration amplitude was kept constant at $0.20 \mathrm{~nm}_{\mathrm{pp}}$ using an amplitude regulator circuit (constantamplitude mode). In FM-AFM, the frequency shift $(\Delta f)$ of $f_{0}$ is detected in the phase-locked loop. The positive and negative $\Delta f$ values of $f_{0}$ are derived from repulsive or attractive forces, respectively. In 2D force mapping, the 2D distribution of $\Delta f$ is measured by tip scanning. The $2 \mathrm{D}$ distribution of $\Delta f$ is theoretically converted to the $2 \mathrm{D}$ force distribution by the Sader and Jarvis method. ${ }^{19}$ In the hydration measurement by FM-AFM, tip scanning speed is adjusted to be sufficiently low compared with the relaxation time of water molecules at the interface, which is checked by measuring the hysteresis of the $F-Z$ curve at the same $X$-coordinate. We used a custom-built controller for scanning and data acquisition.

In our experiment, we cleaved a commercially available muscovite mica substrate in pure water to avoid contamination by room air, and placed the substrate in $1 M \mathrm{KCl}$ aqueous solution. $\mathrm{KCl}$ is necessary for controlling Debye length from the muscovite mica surface.

In another experiment, we prepared a bR sample as follows. At first, $10 \mu \mathrm{l}$ of $20 \mu \mathrm{M}$ bR suspension was added to $490 \mu \mathrm{l}$ of preparation buffer $(10 \mathrm{mM}$ Phosphate Buffer Saline (PBS), $300 \mathrm{mM} \mathrm{KCl}$, and $p \mathrm{H}=7.4)$. After cleaving the mica substrate in pure water, we dropped the above diluted bR suspension onto the mica substrate and left it to stand for about $1 \mathrm{~h}$ until the $\mathrm{bR}$ membrane attached to the mica substrate. After these procedures, we rinsed the substrate with the preparation buffer to remove bR membranes unattached onto the mica substrate, and imaged it in buffer solution (10 mM PBS, $500 \mathrm{mM} \mathrm{KCl}$, and $p \mathrm{H}=7.4$ ).

\section{B. Hydration structure calculation by 3D-RISM}

In order to evaluate the hydration measurement using force detection, we compared the force distribution with the result of the 3D-RISM theory, which is the most advanced theory of molecular liquids based on statistical mechanics. A detailed description of the theory is given in previous papers. ${ }^{4,20,21}$ The calculation was carried out for a doublelayer muscovite in pure water at a temperature of $300 \mathrm{~K}$ with a number density of $0.033313 \AA^{-3}$. The $3 \mathrm{D}$ atomic coordinates of muscovite were taken from an equilibrated structure obtained from MD simulation. ${ }^{22}$ The interatomic interaction parameters were taken from the Clay Force Field (CLAYFF) ${ }^{23}$ The partially linearized hypernetted chain approximation, ${ }^{4}$ also known as the Kovalenko-Hirata (KH) closure, was adopted in the calculation. The 3D-RISM/KH equations were solved on a grid of $64 \times 64 \times 1024$ points in a cubic supercell of $17.9353 \times 20.7320 \times 204.8000 \AA^{3}$ dimensions under a periodic boundary condition. In the theoretical calculation, the existence of the tip was not taken into account.

\section{RESULTS AND DISCUSSION}

\section{A. 2D force mapping by FM-AFM}

Figure 2 shows the experimental result. Figure 2(a) shows an illustration of the model of the cleaved muscovite mica surface and an AFM image of the muscovite mica surface in $\mathrm{KCl}$ aqueous solution. Figure 2(b) shows the experimental result of the imaging of cross-sectional hydration structure in the vertical direction against the mica surface, 


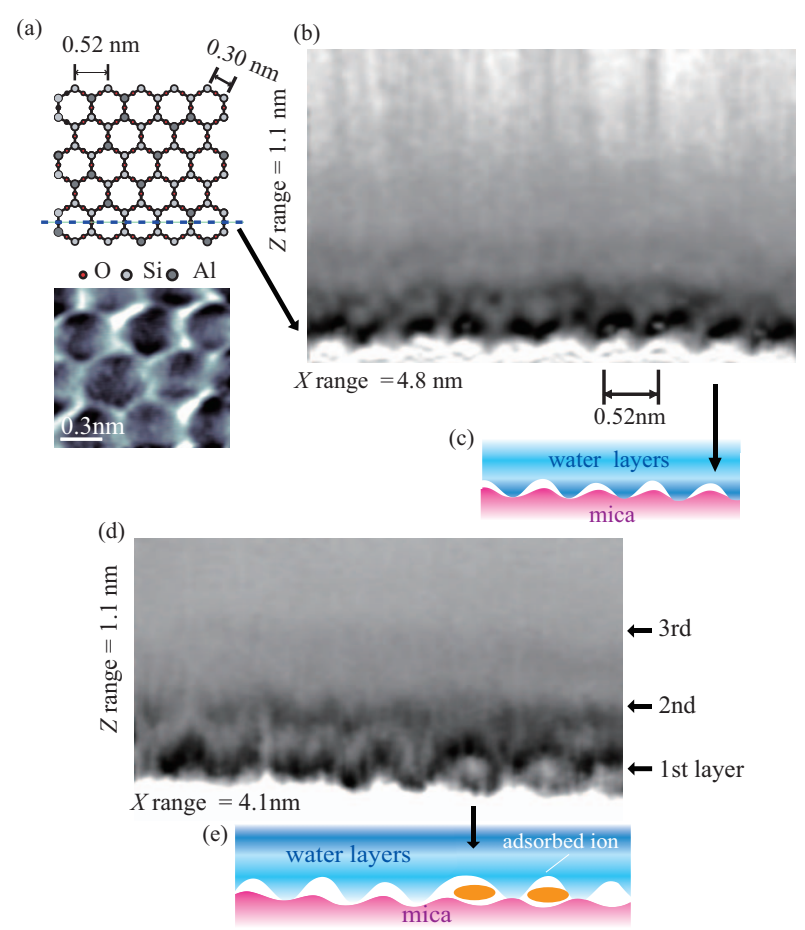

FIG. 2. (a) Schematic illustration of muscovite mica (001) surface structure and FM-AFM image of muscovite mica in water. Hexagonal structures are clearly observed. (b) 2D hydration structure measured by FM-AFM. Periodic structures at the muscovite mica surface and water molecule layers above the surface line, schematically shown by the blue dotted line in Fig. 2(a), are clearly imaged. At the muscovite mica/water interface, the water molecule layer penetrates into the center of the hexagon schematically illustrated in Fig. 2(c), and its penetration has the same periodicity as muscovite mica. In the upper layers, fluid water molecule layers are observed and they show a smaller correlation with the surface structure. (d) 2D hydration structure measured by FM-AFM in another area. In some hollow areas (center of hexagon on tetrahedral sheet, which is a cleaved mica surface), the water molecule layer penetrates the center of the hexagon, and in other hollow areas, white elliptical white contrasts surrounded by dark contrasts, schematically illustrated in Fig. 2(e), are imaged. The contrast is likely to be a $\mathrm{K}^{+}$ion or a hydrated $\mathrm{K}^{+}$ion complex adsorbed on the surface.

which is schematically shown in Fig. 1. In Fig. 2(b), the longitudinal direction of the figure is the $Z$-direction perpendicular to the surface, and the transverse direction is the $X$-direction on the surface shown in Fig. 1. The AFM tip was scanned $4.8 \mathrm{~nm}$ in the $X$-direction and $1.1 \mathrm{~nm}$ in the $Z$-direction. The image contrast indicates the magnitude of the frequency shift $(\Delta f)$ of cantilever mechanical resonance frequency $\left(f_{0}\right)$. The bright and dark contrasts reflect the positive and negative $\Delta f$ values, which reflect repulsive and attractive forces, respectively. A bright area at the bottom of the image indicates a strong repulsive force from the muscovite mica surface, and a $0.52 \mathrm{~nm}$ periodic bright contrast corresponds to the muscovite mica surface area shown by the dotted blue line in Fig. 2(a).

The interaction force applied to the AFM tip is also induced by hydration structures. In the vicinity of the surface periodic white contrast, there is a periodic gray contrast located at the valley of the white contrast, which is schematically shown by the blue area in Fig. 2(c). At the mica surface, hexagonal structures are partially electrically charged because $\mathrm{Si}$ atoms of $\mathrm{SiO}_{4}$ in the basal mica plane are replaced by $\mathrm{Al}$ atoms at a ratio of 3:1 in the bulk. Water mol- ecules interact with negative charges. Therefore, the gray contrast in Fig. 2(b) indicates the existence of water molecules in the hollow area, which is the center of the hexagon.

Figure 2(d) shows another experimental result of 2D hydration measurement in an area different from that shown in Fig. 2(b). The measured area is the dotted blue line area in Fig. 2(a). In this image, in the first water layer indicated by the arrow, the hydration structure is not uniform in the surface direction. This image is schematically shown in Fig. 2(e). In Fig. 2(d), two elliptical white contrasts are found at the center of the two hollow areas, which is schematically shown by the orange ellipse in Fig. 2(e). The origin of these site-specific contrasts is probably the configuration of potassium ions on negatively charged hexagons at the mica surface. In the second layer indicated by the arrow, there is an inhomogeneous density distribution of water molecules in the surface direction. This nonuniformity arises from the inhomogeneous charge distribution. In Fig. 2(d), we can see a weaker dependence of water molecule density on the type of crystal site in the third layer than in the second or first layer.

\section{B. Comparison between FM-AFM and 3D-RISM}

Figure 3 shows a comparison between the force $(F)$ profile obtained by AFM and the profile of the gradient of water molecule density distribution $(-d g / d Z)$, which is calculated from the water molecule density obtained by 3D-RISM. The comparison between force and $-d g / d Z$ is justified as follows. When the average two-body interaction between an AFM tip and a water molecule is given as $u(Z-r)$ (Z: tip position; $r$ : water molecule position), the total potential energy $[U(Z)]$ between the AFM tip and the surrounding water molecules is described as $U(Z)=\int \rho g(r) u(Z-r) d r$, where $\rho$ is the bulk density of water molecules. The integration takes the contribution of all water molecules around the AFM tip. Since $u(Z-r)$ is assumed to be a delta function, $U(Z)$ is proportional to $g(Z)$ and the force applied to the AFM tip $(F)$ is proportional to $-d g / d Z$.

Figure 3(a) shows $F$ - $Z$ curves on the $\mathrm{Si}$ or $\mathrm{Al}$ site and the center of the hexagon shown in Fig. 2(d), aligned with that averaged in the whole area in Fig. 2(d). All curves have long-range attractive forces. This background force reflects the integration of long range van der Waals forces around the tip volume, and has no site specificity. At 0.7 and $1.0 \mathrm{~nm}$ in the $Z$-distance axis, all curves have peaks that have the same force. The less site-specific feature reflects a water molecule configuration determined by the weak interaction between water molecules and the bulk solid surface. Around $0.4 \mathrm{~nm}$, the peak or inflection positions of the three curves differ from each other. Figure 3(b) shows a comparison with the $X Y$-averaged density gradients obtained by 3D-RISM, which is used to calibrate the $F-Z$ profiles. (Details are given in the Appendix.)

Figures 3(c) and 3(d) show a comparison of the force profiles above the $\mathrm{Si}$ or $\mathrm{Al}$ atom and the center of the hexagon, respectively, where potassium ions do not attach, along with the corresponding theoretical density gradient profiles. In the theoretical calculation, no potassium ions are included in the aqueous phase. It is clear that the positions of the first 
(a) Site-specific force profile

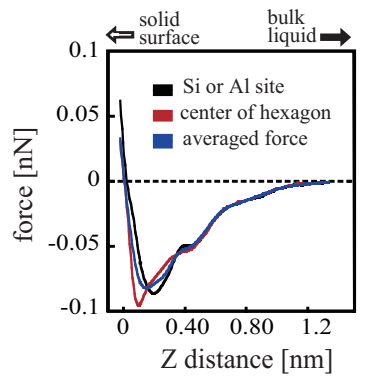

(b) Averaged force profile and gradient of density profile

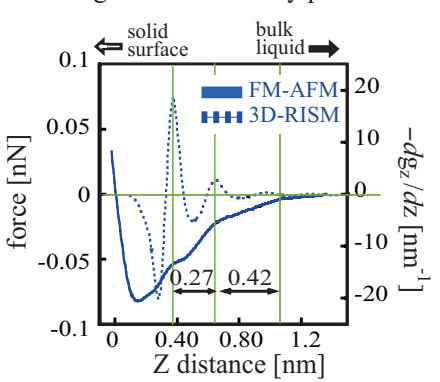

(c) Si or Al site

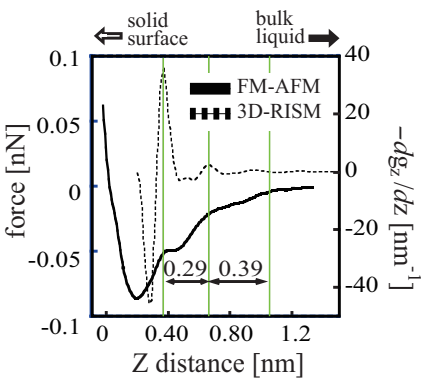

(d) Center of hexagon

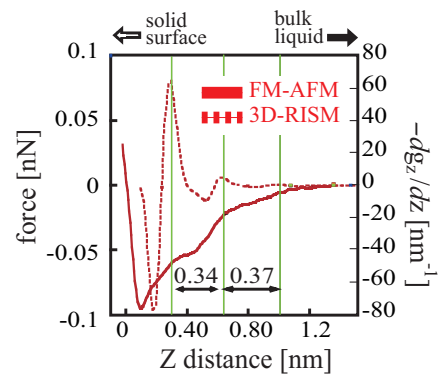

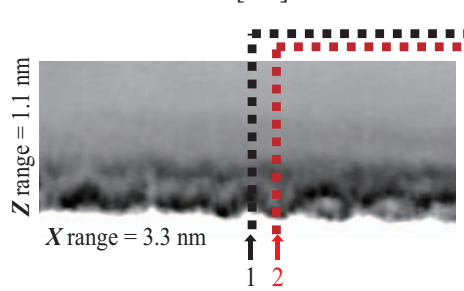

FIG. 3. (a) Site-specific force profiles extracted from Fig. 2(d). Near the solid surface, each curve shows a different value. This characteristic indicates the existence of a hydration layer that is strongly dependent on crystal site. (b) This comparison is used in adjusting the starting point and $Z$-scale in the $F$ - $Z$ and $(-d g / d Z)-Z$ curves. Details are described in the Appendix. (c) Comparison between $F-Z$ and $(-d g / d Z)-Z$ curves on $\mathrm{Si}$ and $\mathrm{Al}$ atoms. There are good correspondences of $Z$-coordinates at the peaks or inflection points. (d) Comparison between local $F-Z$ and $(-d g / d Z)-Z$ curves at the center of hexagon. There are good correspondences between $Z$-coordinates at the second and third peaks or inflection points.

and second inflection points found in the force profile have a good correspondence to those found in the density gradient profiles. The absolute value of force shows no correspondence with the gradient of water molecule density because background long-range force depends on tip shape, and it also shows no crystal site dependence. Thus, the site-specific force profiles obtained at the muscovite mica/ $1 M \mathrm{KCl}$ aqueous solution interface correlate with the gradient of water density profiles.

\section{Application to biomolecules}

As a typical application to studying the structure of biomolecules, we show an experimental result of the 2D hydration measurement of a bR protein membrane. In the archaeobacterium Halobacterium salinarum, bR forms a hexagonal crystal structure in the cell membrane, as shown in Fig. 4(a) and has a lattice spacing of $6.2 \mathrm{~nm}$. Its precise structure, on the atomic scale, has been measured by electron and/or x-ray diffraction analysis. A bR monomer consists of seven $\alpha$-helixes, with three monomers forming a trimer. In the purple membrane, the bR membrane has two sides: the cytoplasmic and extracellular sides. In this study, we investigated the hydration structure on the cytoplasmic side. Figure 4(b) shows the result of the 2D hydration measurement. At the bottom of the image, a lattice spacing of about $6.3 \mathrm{~nm}$ is observed. At the protein/buffer solution interface, we found three quasistructural water molecule layers whose intervals are $0.11,0.14$, and $0.20 \mathrm{~nm}$ in this order from the solid surface. Under the first layer, a low-density region of water molecules, which is shown as a dark-contrast region above the surface white contrast, uniformly spreads over the entire area. Moreover, the interval between layers increases in the upper area from the surface, which reflects a weak interaction with the solid surface and a short correlation time of water molecules in the upper layer.

\section{CONCLUDING REMARKS}

We have succeeded in visualizing 2D cross-sectional hydration structures on the atomic or molecular scale at the muscovite mica/ $1 M \mathrm{KCl}$ aqueous solution interface and $\mathrm{bR}$ membrane/buffer solution interface. In our experimental results, we found that the type of hydration structure in the first layer strongly depends on the surface atom or molecule configuration and that the correlation with the type of surface crystal structure is smaller in the second and third layers than in the first layer. A comparison between FM-AFM and 3DRISM profiles revealed that AFM force profiles are related to naturally established hydration structures at the solid/liquid

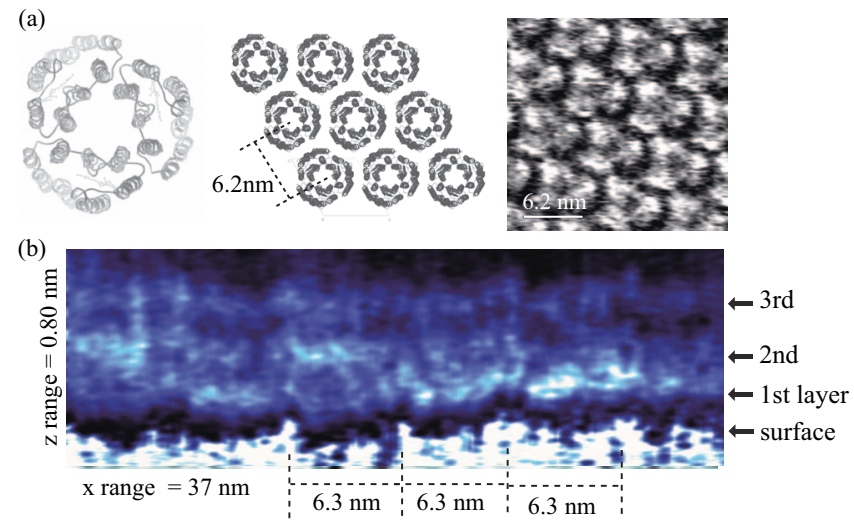

FIG. 4. (a) The left image shows a quaternary structure of bR. A bR molecule forms a tertiary structure where seven transmembrane alpha helixes line up in the order of the primary structure. The quaternary structure (trimer structure) is composed of three tertiary structures. In the membrane, the trimer forms a 2D hexagonal crystal structure shown at the center of Fig. 4(a). The right image in Fig. 4(a) is a FM-AFM image of the 2D hexagonal crystal structure on the cytoplasmic side. (b) 2D hydration structure measured by FM-AFM. Three structurized water molecule layers are clearly observed. The intervals between layers are larger in the upper area, and this result reflects weaker interaction forces between the solid surface and the water molecules at outer layers. 
interface. A more precise description between the force and the density including the effects of tip size and shape should be studied as a future work, and a precise conversion algorithm from force to density is essential for more quantitative hydration measurement.

\section{ACKNOWLEDGMENTS}

T.I. is grateful to Professor A. Kovalenko and Professor F. Hirata for providing him with the program code for the 3D-RISM calculation, which they developed in their earlier studies (Refs. 4, 20, and 21) This work was supported by the Development of Systems and Technology for Advanced Measurement and Analysis of Japan Science and Technology Corporation.

\section{APPENDIX: CALIBRATION OF Z-AXIS BETWEEN AFM DATA AND 3D-RISM DATA}

In general, tip-sample distance defined by an AFM scanner includes systematic error. One of the major reasons for this distance error is the thermal expansion or shrinkage of various mechanical parts of the AFM head. This error is often observed as a 3D topographic distortion in AFM. Therefore, tip-sample distance including this error should be calibrated using a $Z$-length standard. As for the $X$ - and $Y$-directions, we can calibrate the $X$ - and $Y$-scales by considering crystal lattice interval, which is determined by crystal diffraction analysis. However, it is generally difficult to calibrate the $Z$-scale because the imaging area does not always have a $Z$-length standard such as a single-atom step. In particular, in liquid environments, the step edge on a dissoluble crystal is not always observed because its structural stability strongly depends on the physical and chemical properties of both solid and liquid. Therefore, in our solvation measurements, we adopted a theoretically calculated density profile of liquid molecules as a Z-length standard.

Here, we show how we calibrated the $Z$-scale of the AFM scanner in this study. When we measure the tip-sample distance $(Z)$ dependence of applied force $(F)$ toward the tip at the solid/liquid interface, the obtained force-distance curve $(F-Z)$ is oscillating. In the principle of solvation measurement by AFM, $F$ is approximately proportional to the density gradient of solvent molecules. The AFM tip senses a repulsive force at a local maximum position of the $(-d g / d Z)-Z$ curve, and correspondingly the $F-Z$ curve has a local maximum or an inflection point. Therefore, if we have a reference profile of the $(-d g / d Z)-Z$ curve as a $Z$-length standard, we can calibrate the $Z$-scale of the AFM scanner by comparing the specific interval in the oscillating $F-Z$ curve with the corresponding interval in the reference profile. In this study, we adopted the $X Y$-averaged $(-d g / d Z)-Z$ curve calculated using the 3D-RISM theory as a reference profile.

The global van-der-Waals-force-like behavior found in the $F-Z$ curve apparently originates from the interaction between the solid surface and the whole area of the AFM tip, whereas additional peaks or inflection points to the global curve represent solvent-molecule layers. Thus, the rapid increase on the far left indicates a repulsive force from the solid surface, and the first small peak on the global attractive force curve corresponds to the first solvation peak.

On the other hand, the upstroke of the $(-d g / d Z)-Z$ curve around the $Z$-origin is ascribed to the direct repulsive interaction between solvent water molecules and the solid surface, while the next large peak is assigned to the first solvation peak. Since the source of the large force around the $Z$-origin in the $F-Z$ curve is different from that in the $(-d g / d Z)-Z$ curve, it is difficult to set the $Z$-origin by superposition in this region. Thus, we adjusted the $Z$-origin by the superposition of the first solvation peaks in the $F-Z$ and $(-d g / d Z)-Z$ curves. Then, we scaled the interval between the first and second solvation peaks in the $F-Z$ curve so that it became equal to the corresponding interval in the $(-d g / d Z)-Z$ curve.

The adjustment was carried out only for the $X Y$-averaged profile [Fig. 3(b)], and the determined calibrations were applied to the site-specific profiles [Figs. 3(c) and $3(d)]$ without modification. Note that the agreements between the first and second peak positions of the site-specific $F-Z$ and $(-d g / d Z)-Z$ profiles are therefore not an artifact of this adjustment but imply that the $F-Z$ profiles reflect the site-specific solvation structure as the $(-d g / d Z)-Z$ profiles do.

${ }^{1}$ I. A. Novikov, A. I. Ivannikov, V. S. Kobelev, and V. Y. A. Volkov, Mol. Biol. 19, 1029 (1985).

${ }^{2}$ J. A. Rupley and G. Careri, Adv. Protein Chem. 41, 37 (1991).

${ }^{3}$ R. Akiyama and F. Hirata, J. Chem. Phys. 108, 4904 (1998).

${ }^{4}$ A. Kovalenko and F. Hirata, J. Chem. Phys. 110, 10095 (1999).

${ }^{5}$ S. P. Jarvis, T. Uchihashi, T. Ishida, Y. Nakayama, and H. Tokumoto, J. Phys. Chem. B 104, 6091 (2000).

${ }^{6}$ T. Fukuma, K. Kobayashi, K. Matsushige, and H. Yamada, Appl. Phys. Lett. 86, 193108 (2005).

${ }^{7}$ T. Fukuma, M. J. Higgins, and S. P. Jarvis, Biophys. J. 92, 3603 (2007).

${ }^{8}$ W. Hofbauer, R. J. Ho, R. Hairulnizam, N. N. Gosvami, and S. J. O'Shea, Phys. Rev. B 80, 134104 (2009).

${ }^{9}$ K. Kimura, S. Ido, N. Oyabu, K. Kobayashi, T. Imai, and H. Yamada, Ext. Abstr. 11th International Conference on Non-Contact Atomic Force Microscopy, 2008, p. 62.

${ }^{10}$ G. R. Edwards, L. F. Evans, and A. F. Zipper, Trans. Faraday Soc. 66, 220 (1970).

${ }^{11}$ J. L. Caslavsky and K. Vedam, J. Appl. Phys. 42, 516 (1971).

${ }^{12}$ J. Hu, X.-D. Xiao, D. F. Ogletree, and M. Salmeron, Science 268, 267 (1995).

${ }^{13}$ J. Hu, X.-D. Xiao, D. F. Ogletree, and M. Salmeron, Surf. Sci. 344, 221 (1995).

${ }^{14}$ P. B. Miranda, L. Xu, Y. R. Shen, and M. Salmeron, Phys. Rev. Lett. 81, 5876 (1998).

${ }^{15}$ M. Odelius, M. Bernasconi, and M. Parrinello, Phys. Rev. Lett. 78, 2855 (1997).

${ }^{16}$ L. Cheng, P. Fenter, K. L. Nagy, M. L. Schlegel, and N. C. Sturchio, Phys. Rev. Lett. 87, 156103 (2001).

${ }^{17}$ S. Park and G. Sposito, Phys. Rev. Lett. 89, 085501 (2002).

${ }^{18}$ K. Kobayashi, H. Yamada, H. Itoh, T. Horiuchi, and K. Matsushige, Rev. Sci. Instrum. 72, 4383 (2001).

${ }^{19}$ J. E. Sader and S. P. Jarvis, Appl. Phys. Lett. 84, 1801 (2004).

${ }^{20}$ A. Kovalenko, in Molecular Theory of Solvation, edited by F. Hirata (Kluwer, Dordrecht, 2003), pp. 169-275.

${ }^{21}$ A. Kovalenko and F. Hirata, J. Chem. Phys. 112, 10391 (2000).

${ }^{22}$ J. Wang, A. G. Kalinichev, R. J. Kirkpatrick, and R. T. Cygan, J. Phys. Chem. B 109, 15893 (2005).

${ }^{23}$ R. T. Cygan, J.-J. Liang, and A. G. Kalinichev, J. Phys. Chem. B 108, 1255 (2004). 